\title{
Pitfalls and problems encountered in rat model sciatic nerve surgery
}

\author{
Marin Andrei ${ }^{1}$, Mihai Ruxandra loana ${ }^{1}$, \\ Marin Georgiana Gabriela ${ }^{2}$
}

\author{
1 Plastic Surgery Department, "Bagdasar Arseni" Emergency Hospital, \\ Bucharest, ROMANIA \\ ${ }^{2}$ Cardiology Department, "CC Iliescu" Hospital, Bucharest, RomAnIA
}

\begin{abstract}
When learning the basics of microsurgery, a trainee must be equipped with patience and perseverance in order to evolve. One must have the ground knowledge when it comes to peripheral nerve injury and nerve regeneration process in order to fully understand that the technique is vital for the outcome and final results. Furthermore, a trainee must practice on non-living tissue before performing successful in vivo operations and even in this case, one may be confronted with problems regarding the surgical technique. [1] The following article aims to reveal the main problems/mistakes when performing sciatic nerve surgery in an in vivo experimental model and the solutions for these problems.
\end{abstract}

\section{INTRODUCTION}

There are many experimental surgical projects performed on the sciatic nerve of the rat. [2] There are many reasons why researchers choose this model: high similitude in nerve regeneration between the rat and the human; the lab rat (most commonly used the Wistar rat) is more available compared to other species and easier to manipulate; the anatomy of the sciatic nerve of the rat offers easy access, without significant comorbidity; the diameter and the length of the nerve permits different types of experiments (direct suture, reconstruction of different gaps with nerve conducts). [3] The sciatic nerve has a length of $\sim 2,5-3 \mathrm{~cm}$ before it divides into 3 terminal branches - common peroneal nerve, tibial nerve and sural nerve.

Although rat's sciatic nerve is one of the most used models for nerve regeneration it still may cause difficulties for an inexperienced surgeon. The problems one may encounter are related to anesthesia, [4] rat anatomy knowledge or microsurgical technique and each one of these issues may cause an enormous impact on the final outcome. Understanding the theoretical aspects of nerve regeneration [5],[6],[7] may help improve the surgical technique, especially when it comes to atraumatic manipulation.

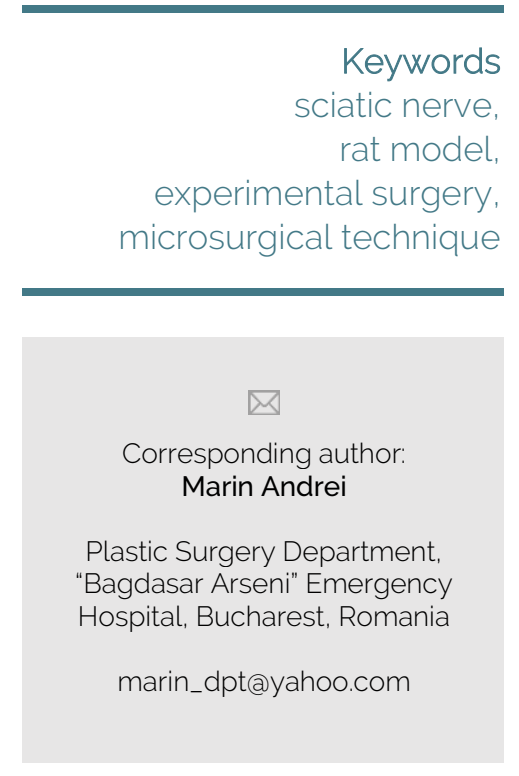

Copyright and usage. This is an Open Access article, distributed under the terms of the Creative Commons Attribution Non-Commercial No Derivatives License (https://creativecommons org/licenses/by-nc-nd/4.0/) which permits noncommercial re-use, distribution, and reproduction in any medium, provided the original work is unaltered and is properly cited

The written permission of the Romanian Society of Neurosurgery must be obtained for commercial re-use or in order to create a derivative work.

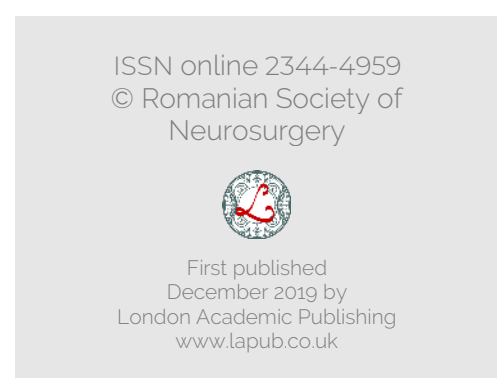




\section{MATERIAL AND METHOD}

For this review there were used Wistar rats in different microsurgery training laboratories. The rats varied in age and weight (between 250-350g or 7-9 weeks). The anesthesia protocol consisted of a mixture of ketamine $(75 \mathrm{mg} / \mathrm{kg})$ and xylazine $(10 \mathrm{mg} / \mathrm{kg})$ administered intraperitoneally. A precise scale was used to weigh every animal before administrating the anesthesia. There were used proper microsurgical instruments as well as a microscope and appropriate sutures (8-0,9-0,10-0).

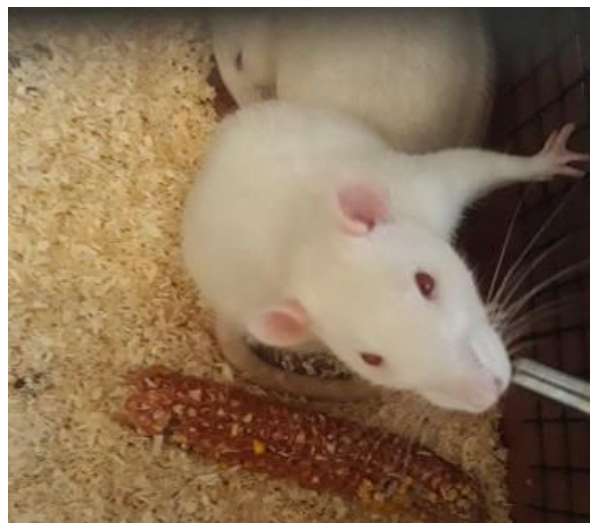

Wistar rat

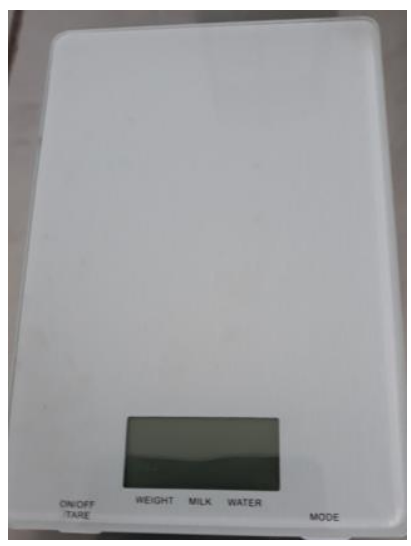

Scale

Microsurgical instruments

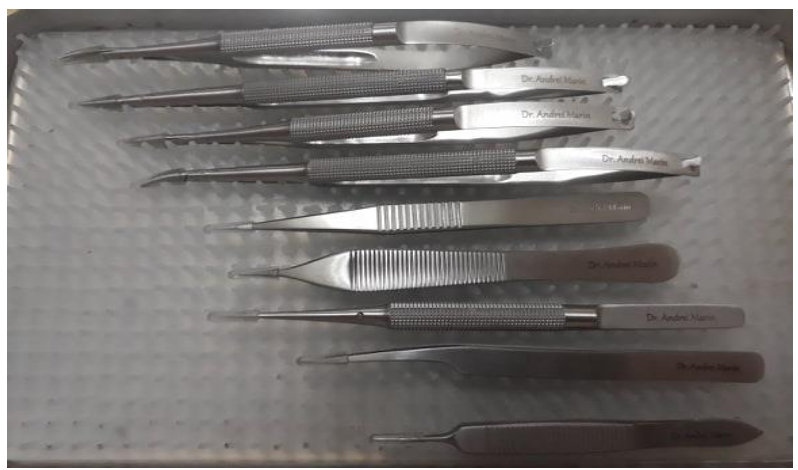

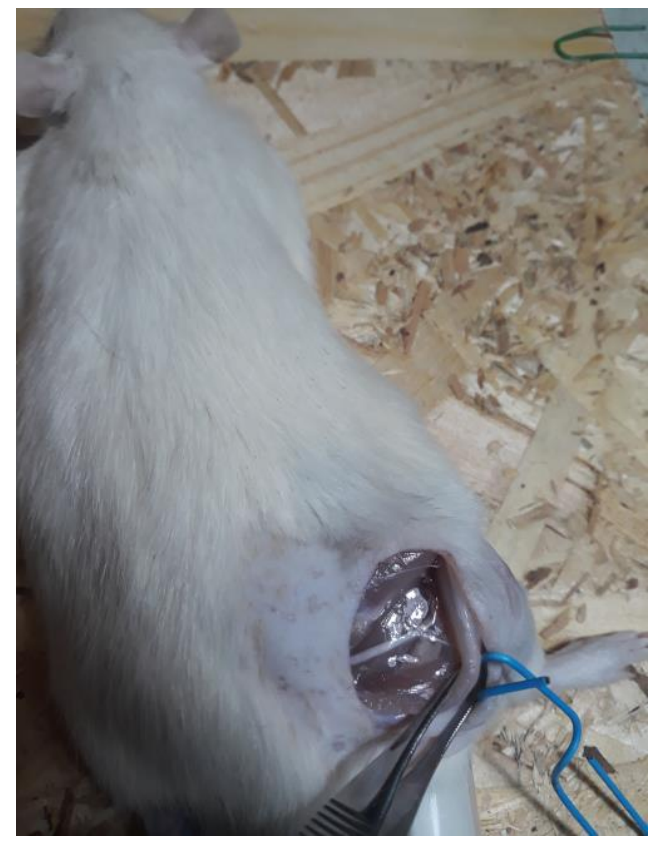

Sciatic nerve exposure

After shaving the dorsal aspect of the gluteal and thigh region, the rat is placed in prone position. An oblique-transverse skin incision is made and dissection along the biceps muscle fibers is performed in order to expose the underlying sciatic nerve. Mehedintu et al described different types of incisions to access the sciatic nerve. [7],[8] After nerve exposure, the rest of the operation is done under the microscope. This involves nerve dissection, nerve section and subsequent nerve repair. Proximal to the its division, the sciatic nerve can be used for various operations (direct suture after section, autografting, using different nerve conducts for iatrogenic nerve defects).

\section{RESULTS}

A frequent mistake of the in vivo experiment is the incorrect administration of the anesthesia, which must be very well dosed and not lead to to overdosing and rat death.

Aside from the anesthetic issues, the most frequent problems when repairing a nerve defect with autograft arise from lack of experience. [9] These are: identifying the sciatic nerve correctly, improper incision site, excessive nerve dissection for a better exposure, choosing an unsuitable location to create the nerve gap, as well as microsurgical technique errors (inappropriate cooptation, high suture tension). Inadequate sutures for the nerve size also influence the recovery results. 


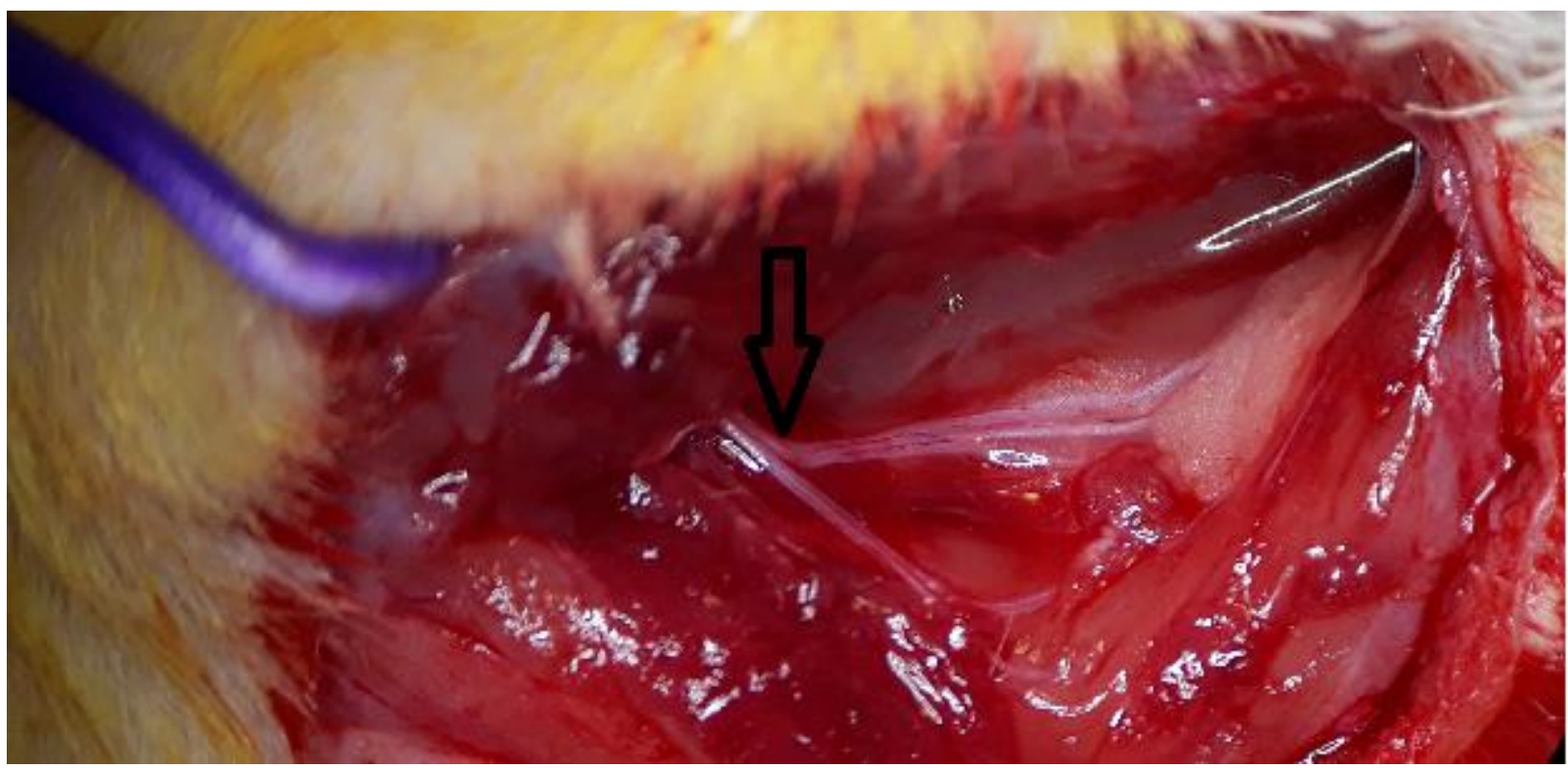

Distal incision beyond the division of the sciatic nerve (marked with arrow)

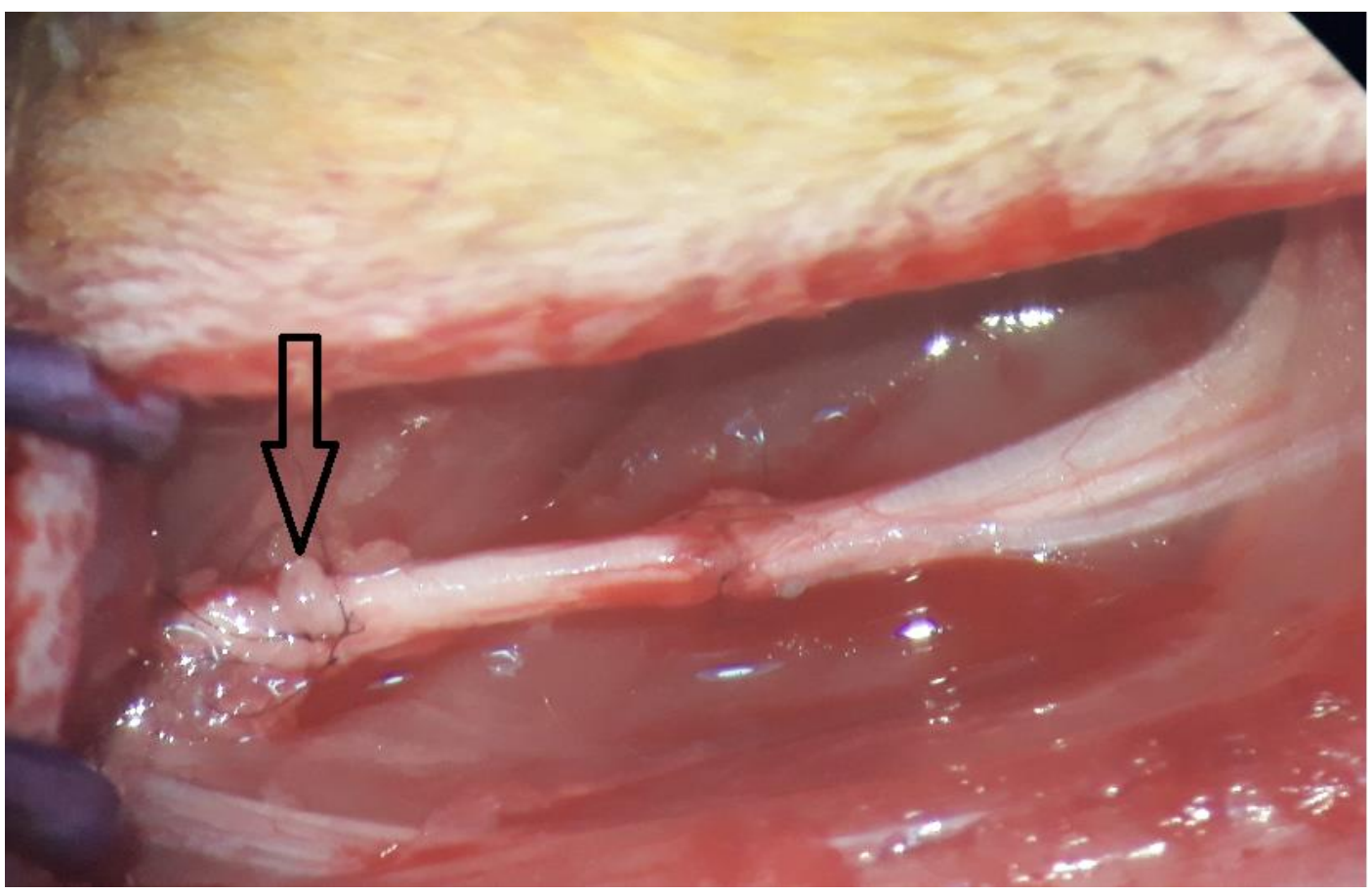

Axoplasmatic protrusion (arrow) in the proximal nerve suture due to insufficient proximal dissection

Identifying the sciatic nerve correctly poses no problem if the skin incision site and the muscle dissection is performed in the right area and in a proper plane. A distal incision (too lateral and caudal), however, may create confusion in case not all branches are visible, thus mistaking the sciatic nerve to one of its branches. A contributing factor to this problem is represented by the placing of the rat in a slight prone-lateral position (instead of the custom prone position). These branches are half or even $1 / 3$ of the sciatic nerve diameter $(\sim 1 \mathrm{~mm})$. To prevent this situation, the skin incision should be placed more cranial and medial, parallel and $1 \mathrm{~cm}$ inferior to the femur, with the rat in prone position. When suspecting that the first incision is inappropriate, with exposure of just one branch of the sciatic nerve, extending the incision more proximal can solve the problem by identifying the correct nerve.

Another technical error is creating the nerve gap 
too proximal (or not dissecting enough of the sciatic nerve from the muscle tissue in the proximal part). After nerve sectioning, the elastic fibers in the structure of the nerve determine retraction of the proximal stump in a plane which is hard to access, thus making the nerve suture difficult to perform. In order to prevent this problem, a proper dissection and the selection of the central area of the sciatic nerve to create the gap is recommended. The consequences of suturing a retracted stump are: inappropriate traction of the nerve (which risks injuring the nerve fascicles), unmatched fascicles in the suture (the surgeon is no longer able to observe the epineural vessels to guide a correct cooptation), axoplasmatic protrusion due to excessive traction and uneven suture. In all cases, inappropriate dissection results in prolonged operating times.

Creating the nerve gap too distal (close to the division of the sciatic nerve) poses a problem for nerve suturing because an epineural suture tends to shred the common sheath of the branches, dividing them into 3 separate nerves (which require individual repair). The ideal distal sectioning site is $\sim 0,7 \mathrm{~cm}$ proximal to the visible division of the sciatic nerve in order to have enough connective tissue for a strong nerve suture.

\section{DiscusSiON}

It is recommended not to operate on rats lighter than $250 \mathrm{~g}$ and younger than 7 weeks of age. Otherwise the nerve diameter might be smaller than $2 \mathrm{~mm}$, requiring adequate microsurgery equipment as well as a greater experience in nerve surgery. Above $350 \mathrm{~g}$ or 9 weeks of age, the animals require more substance to induce anesthesia, without any extra benefits.

Multiple administration of anesthesia might lead to overdose, causing respiratory distress and in the end exitus. For this reason, if the anesthesia doesn't work accordingly from the beginning with the calculated dose, the operation should be postponed and the anesthetic options should be revised.

The iatrogenic nerve gap should be placed centrally, after exposing the sciatic nerve from its origin to its division.

\section{CONCLUSIONS}

Microsurgery requires adequate instruments and special training. Calculating the exact quantity of anesthetic reduces the death risk. Experience and attention are key factors in avoiding different complications related to nerve surgery. The microsurgical technique involves an atraumatic manipulation of the tissue, correct cooptation without suture tension and right alignment of the nerve fascicles. Respecting these rules results in a good nerve regeneration, with positive clinical outcomes.

\section{REFERENCES}

1. Christensen TJ, Anding W, Shin AY, Bishop AT, Moran SL.The Influence of Microsurgical Training on the Practice of Hand Surgeons. J Reconstr Microsurg. 2015 Jul;31(6):442-9. doi: 10.1055/s-0035-1549443. Epub 2015 Apr 20.

2. Andrei Marin, Georgiana Gabriela Marin, Anca Patea, Dan Mircea Enescu. Timing, Logistics and Bureaucratic Process in Planning an Experimental In Vivo Nerve Regeneration Project from A to Z. Modern Medicine | 2019, Vol. 26, No. 2

3. Marin Andrei, Marin Georgiana Gabriela, Dobrete Nicoleta Amalia, Enescu Dan Mircea. Learning curve in rat dissection for experimental sciatic nerve repair. Romanian Neurosurgery (2019) XXXIII (3) September 2019. www.journals.lapub.co.uk/index.php/roneurosurgery

4. Costea Ruxandra, Daniel Lastofka, and Mihai Mehedinţu. "Comparison of Ketamine-Medetomidine-butorphanol and Ketamine-dexmedetomidine-butorphanol Anesthesia in Rats." Agriculture and Agricultural Science Procedia 6 (2015): 305-308.

5. Lastofka, D., Manolescu, N., Balint, E., Costea, R., \& Ionescu, M. M. (2017). Histopathological modifications in sciatic nerve allograph in Wistar rats. Journal of Biotechnology, 256, S86.

6. Scholz T, Krichevsky A, Sumarto A, et al. Peripheral nerve injuries: an international survey of current treatments and future perspectives. J Reconstr Microsurg 2009;25(6):33944

7. Andrei, M., loana, M., \& Mircea, E. (2019). Underlying histopathology of peripheral nerve injury and the classical nerve repair techniques. Romanian Neurosurgery, 33(1), 17-22. https://doi.org/10.33962/roneuro-2019-003

8. Mihai Mehedintu-Ionescu, Ovidiu Stefanescu, Radu Cristian Jecan. Nervous Regeneration Allograph Type of Periferic Nerv. Modern Medicine | 2019, Vol. 26, No. 2

9. Ionescu, M. M., Costea, R., Balint, E., \& Lastofka, D. (2017). Surgery technique models of sciatic nerve allograph in Wistar rats. Abstracts/Journal of Biotechnology 256S, 44, S116

10. Kuffler DP. An assesment of current techniques for inducing axon regeneration and neurological reovery following peripheral nerve trauma. Prog Neurobiol 2014;116:1-12. 